\title{
Variability in reference evapotranspiration and moisture availability in three agroclimatic zones of Punjab, India
}

\author{
S. AATRALARASI ${ }^{1}$, P.K.KINGRA ${ }^{1 *}$, R.K.SETIA ${ }^{2}$ and SOM PAL SINGH ${ }^{1}$ \\ ${ }^{1}$ Dept. of Climate Change and Agricultural Meteorology, PAU, Ludhiana \\ ${ }^{2}$ Punjab Remote Sensing Centre, Ludhiana, India \\ *Corresponding author : pkkingra@pau.edu
}

\begin{abstract}
A research study was undertaken to estimate reference evapotranspiration $\left(E T_{0}\right)$, moisture index (MI) and aridity index $(\mathrm{Al})$ and analyse their trends in different locations/zones of Punjab. Annual and seasonal ET, was observed to be highest in south-west and lowest in north-east zone. North-east zone experienced increasing trend in annual as well as seasonal ET. Districts within the central zone had large variability within the zone. MI and Al were in the order of north-east zone followed by Central zone and South-west zone indicating better moisture availability conditions in north-east. Mean difference in annual and seasonal $\mathrm{MI}$ and $\mathrm{Al}$ also indicated statistical similarities within the districts of north-east and south-west zones but different from other zones. Thus, increasing trend of $\mathrm{ET}_{\mathrm{O}}$ in north-east region and higher $\mathrm{ET}_{\mathrm{O}}$ range in south-west zone along with severe moisture availability necessitates the need for more careful planning of water resources in the region.
\end{abstract}

Key words: Reference evapotranspiration, moisture index, aridity index, Punjab

The agriculture sector is the largest consumer of water resources in Punjab. The total water deficit of Punjab is 8.99 Billion Cubic Meters (BCM) annually which will increase to 9.12 BCM by 2020 (Anon, 2017). Reference evapotranspiration $\left(\mathrm{ET}_{\mathrm{O}}\right)$ is a key factor of water balance. $\mathrm{ET}_{\mathrm{O}}$ expends two-third of the terrestrial precipitation (worldwide terrestrial rainfall is $98.5 \times 10^{3} \mathrm{~km}^{3}$ year ${ }^{-1}$, evapotranspiration is $65.5 \times 10^{3} \mathrm{~km}^{3}$ year ${ }^{-1}$ ) (Mao et al. 2015). Reference evapotranspiration can be used for assessment of crop water requirements which is used for maintaining agricultural production and sustaining hydrological cycle. Accurate estimation of $\mathrm{ET}_{\mathrm{O}}$ can be useful for formulating and altering the cropping plans and cultural operations to deal with anomalous weather for improving agricultural production in rainfed regions (Halikatti et al. 2010).

Holzman et al (2013) found that soil moisture has direct relationship with the output of rainfed crops and moisture index helps in prediction of yield even before the harvest of the crop. Kaur and Gill (2017) calculated ET $_{0}$ for present, mid and end century at central Punjab using Papadakis method and found rise in water requirement from 2011-2040 (1027 mm) to 2071-2100 (1150 mm). Under such conditions, a profound understanding of water availability can be obtained by studying $\mathrm{ET}_{\mathrm{O}}$, MI and $\mathrm{AI}$ on spatial and temporal scale. In view of this, the current research aims to study the variability of these parameters between and within different agroclimatic zones of Punjab. Under present scenario of increasing water crisis in the state, this study may help in better crop planning through judicious use of water.

\section{MATERIALS AND METHODS}

\section{Data collection}

The long-term data (1971-2018) of maximum temperature, minimum temperature and rainfall were collected from the Department of Climate Change and Agricultural Meteorology, Punjab Agricultural University (PAU), Ludhiana, Regional Research Stations of PAU and India Meteorological Department. for different districts viz. Gurdaspur and SBS Nagar in north-east region, Amritsar, Ludhiana and Patiala in central region and Firozpur and Bathinda in south-west region of the state.

\section{Computation of reference evapotranspiration (ET $)$}

$\mathrm{ET}_{\mathrm{O}}$ was computed by using the Hargreaves-Samani method (Hargreaves and Samani, 1985) as following:

$$
\mathrm{ET}_{\text {oHS }}=0.0023 \mathrm{R}_{\mathrm{a}}(\mathrm{T}+17.8) \sqrt{\mathrm{T}_{\max }-\mathrm{T}_{\min }}
$$

Where, $\mathrm{ET}_{\mathrm{oHS}}$ is grass reference evapotranspiration $\left(\mathrm{mm} \mathrm{d}^{-1}\right), \mathrm{T}$ is daily mean air temperature $\left({ }^{\circ} \mathrm{C}\right), \mathrm{T}_{\max }$ is daily maximum air temperature $\left({ }^{\circ} \mathrm{C}\right), \mathrm{T}_{\min }$ is daily minimum air temperature $\left({ }^{\circ} \mathrm{C}\right)$ and $\mathrm{R}_{\mathrm{a}}$ is water equivalent of the extra terrestrial radiation $\left(\mathrm{mm} \mathrm{d}^{-1}\right)$. 
Table 1: Average $\mathrm{ET}_{\mathrm{O}}$ in different agroclimatic zones of Punjab (1971 to 2018)

\begin{tabular}{|c|c|c|c|c|c|}
\hline $\begin{array}{l}\text { Agroclimatic } \\
\text { zones / stations }\end{array}$ & Mean \pm SD $(\mathrm{mm})$ & $\mathrm{CV}$ & $\begin{array}{l}\text { Rate of Change } \\
\left(\mathrm{mm} \text { year }{ }^{-1}\right)\end{array}$ & $\mathrm{R}^{2}$ & Trend \\
\hline \multicolumn{6}{|c|}{ Annual ET } \\
\hline \multicolumn{6}{|l|}{ North-east zone } \\
\hline Gurdaspur & $1567.3 \pm 61.70$ & 3.94 & 2.80 & 0.40 & Significant \\
\hline SBS Nagar & $1562.5 \pm 90.40$ & 5.78 & 3.92 & 0.39 & Significant \\
\hline Average & $1564.89 \pm 73.66$ & 4.71 & 3.41 & 0.42 & Significant \\
\hline \multicolumn{6}{|c|}{ Central plain zone } \\
\hline Amritsar & $1681.5 \pm 47.80$ & 2.84 & -0.61 & 0.03 & NS \\
\hline Ludhiana & $1592.3 \pm 54.40$ & 3.42 & -2.55 & 0.43 & Significant \\
\hline Patiala & $1611.5 \pm 58.80$ & 3.65 & 1.27 & 0.10 & Significant \\
\hline Average & $1628.46 \pm 44.53$ & 2.73 & -0.48 & 0.02 & NS \\
\hline \multicolumn{6}{|c|}{ South-western zone } \\
\hline Bathinda & $1732.9 \pm 53.30$ & 3.07 & -1.37 & 0.13 & Significant \\
\hline Firozpur & $1712.9 \pm 40.60$ & 2.37 & 0.85 & 0.09 & Significant \\
\hline Average & $1722.9 \pm 36.94$ & 2.14 & -0.26 & 0.01 & NS \\
\hline \multicolumn{6}{|c|}{${\text { Kharif } \mathrm{ET}_{\mathrm{o}}}$} \\
\hline \multicolumn{6}{|l|}{ North-east zone } \\
\hline Gurdaspur & $997.4 \pm 39.50$ & 3.96 & 1.49 & 0.28 & Significant \\
\hline SBS Nagar & $979 \pm 54.60$ & 5.58 & 2.16 & 0.32 & Significant \\
\hline Average & $988.21 \pm 44.34$ & 4.49 & 1.81 & 0.33 & Significant \\
\hline \multicolumn{6}{|c|}{ Central plain zone } \\
\hline Amritsar & $1056.6 \pm 37.50$ & 3.55 & -0.30 & 0.01 & NS \\
\hline Ludhiana & $982.4 \pm 45.60$ & 4.64 & 2.32 & 0.50 & Significant \\
\hline Patiala & $982.9 \pm 43.30$ & 4.41 & 0.55 & 0.03 & NS \\
\hline Average & $1007.28 \pm 35.00$ & 3.47 & -0.63 & 0.06 & NS \\
\hline \multicolumn{6}{|c|}{ South-western zone } \\
\hline Bathinda & $1078.9 \pm 41.10$ & 3.81 & -0.83 & 0.08 & Significant \\
\hline Firozpur & $1070.9 \pm 33.10$ & 3.09 & 0.64 & 0.07 & NS \\
\hline Average & $1074.9 \pm 29.86$ & 2.78 & -0.09 & 0.00 & NS \\
\hline \multicolumn{6}{|c|}{ Rabi $\mathbf{E T}_{\mathrm{o}}$} \\
\hline \multicolumn{6}{|l|}{ North-east zone } \\
\hline Gurdaspur & $569.3 \pm 29.10$ & 5.11 & 1.41 & 0.44 & Significant \\
\hline SBS Nagar & $583.3 \pm 39.90$ & 6.84 & 1.84 & 0.43 & Significant \\
\hline Average & $576.33 \pm 31.96$ & 5.55 & 1.68 & 0.52 & Significant \\
\hline \multicolumn{6}{|c|}{ Central plain zone } \\
\hline Amritsar & $624.2 \pm 23.50$ & 3.77 & -0.22 & 0.02 & NS \\
\hline Ludhiana & $609.4 \pm 24.90$ & 4.08 & -0.23 & 0.02 & NS \\
\hline Patiala & $628.3 \pm 29.30$ & 4.67 & 0.74 & 0.13 & Significant \\
\hline Average & $620.63 \pm 24.46$ & 3.94 & 0.20 & 0.01 & NS \\
\hline \multicolumn{6}{|c|}{ South-western zone } \\
\hline Bathinda & $653.5 \pm 27.90$ & 4.28 & -0.51 & 0.06 & NS \\
\hline Firozpur & $641.6 \pm 20.30$ & 3.16 & 0.29 & 0.04 & NS \\
\hline Average & $647.54 \pm 21.46$ & 3.31 & -0.11 & 0.01 & NS \\
\hline
\end{tabular}


Table 2: Average MI in different agroclimatic zones of Punjab (1971 to 2018)

\begin{tabular}{|c|c|c|c|c|c|}
\hline $\begin{array}{l}\text { Agroclimatic } \\
\text { zones / stations }\end{array}$ & Mean \pm SD & $\mathrm{CV}$ & $\begin{array}{l}\text { Rate of Change } \\
\text { (units year-1) }\end{array}$ & $\mathrm{R}^{2}$ & Trend \\
\hline \multicolumn{6}{|c|}{ Annual MI } \\
\hline \multicolumn{6}{|l|}{ North-east zone } \\
\hline Gurdaspur & $-35.1 \pm 16.50$ & 47.14 & -0.231 & 0.038 & NS \\
\hline SBS Nagar & $-32.5 \pm 17.80$ & 54.80 & -0.133 & 0.011 & NS \\
\hline Average & $-33.82 \pm 15.68$ & 46.36 & -0.18 & 0.03 & NS \\
\hline \multicolumn{6}{|c|}{ Central plain zone } \\
\hline Amritsar & $-55.1 \pm 14.00$ & 25.48 & 0.008 & 0 & NS \\
\hline Ludhiana & $-52.1 \pm 14.90$ & 28.57 & 0.122 & 0.01 & NS \\
\hline Patiala & $-53.2 \pm 15.90$ & 29.96 & -0.069 & 0.00 & NS \\
\hline Average & $-53.49 \pm 13.15$ & 24.58 & 0.02 & 0.00 & NS \\
\hline \multicolumn{6}{|c|}{ South-western zone } \\
\hline Bathinda & $-74.8 \pm 8.50$ & 11.30 & 0.092 & 0.023 & NS \\
\hline Firozpur & $-73.6 \pm 8.80$ & 11.97 & 0.027 & 0.002 & NS \\
\hline Average & $-74.23 \pm 7.97$ & 10.74 & 0.06 & 0.01 & NS \\
\hline \multicolumn{6}{|c|}{ Kharif MI } \\
\hline \multicolumn{6}{|l|}{ North-east zone } \\
\hline Gurdaspur & $-18.4 \pm 24.60$ & 133.56 & -0.209 & 0.014 & NS \\
\hline SBS Nagar & $-11 \pm 26.20$ & 238.05 & -0.038 & 0 & NS \\
\hline Average & $-14.7 \pm 23.67$ & 161.13 & -0.120 & 0.01 & NS \\
\hline \multicolumn{6}{|c|}{ Central plain zone } \\
\hline Amritsar & $-43.7 \pm 21.90$ & 50.12 & 0.23 & 0.022 & NS \\
\hline Ludhiana & $-35.9 \pm 25.20$ & 70.10 & 0.206 & 0.012 & NS \\
\hline Patiala & $-33.4 \pm 26.00$ & 77.79 & -0.007 & 0.00 & NS \\
\hline Average & $-37.7 \pm 18.82$ & 49.95 & 0.120 & 0.01 & NS \\
\hline \multicolumn{6}{|c|}{ South-western zone } \\
\hline Bathinda & $-66 \pm 12.50$ & 18.95 & 0.166 & 0.034 & NS \\
\hline Firozpur & $-64.3 \pm 12.80$ & 19.92 & 0.078 & 0.01 & NS \\
\hline Average & $-65.15 \pm 11.70$ & 18.05 & 0.120 & 0.02 & NS \\
\hline \multicolumn{6}{|c|}{ Rabi MI } \\
\hline \multicolumn{6}{|l|}{ North-east zone } \\
\hline Gurdaspur & $-63.9 \pm 18.80$ & 29.38 & -0.305 & 0.05 & NS \\
\hline SBS Nagar & $-68.6 \pm 17.00$ & 24.75 & -0.258 & 0.041 & NS \\
\hline Average & $-66.2 \pm 16.51$ & 24.93 & -0.290 & 0.06 & NS \\
\hline \multicolumn{6}{|c|}{ Central plain zone } \\
\hline Amritsar & $-76.3 \pm 14.10$ & 18.47 & -0.063 & 0.004 & NS \\
\hline Ludhiana & $-79 \pm 12.00$ & 15.17 & -0.060 & 0.005 & NS \\
\hline Patiala & $-84.7 \pm 10.70$ & 12.63 & -0.098 & 0.02 & NS \\
\hline Average & $-79.96 \pm 11.01$ & 13.77 & -0.070 & 0.01 & NS \\
\hline \multicolumn{6}{|c|}{ South-western zone } \\
\hline Bathinda & $-89 \pm 5.70$ & 6.40 & -0.046 & 0.012 & NS \\
\hline Firozpur & $-89 \pm 5.60$ & 6.24 & -0.092 & 0.052 & NS \\
\hline Average & $-89 \pm 5.25$ & 5.90 & -0.070 & 0.03 & NS \\
\hline
\end{tabular}




\section{Computation of moisture index (MI)}

The availability of moisture for sufficient and efficient crop production was calculated by moisture index (Krishnan and Singh, 1972) as following:

Moisture Index $(\mathrm{MI})=\frac{\mathrm{P}-\mathrm{ET}_{\mathrm{O}}}{\mathrm{ET}_{\mathrm{O}}}$

Where, $\mathrm{P}$ is precipitation and $\mathrm{ET}_{\mathrm{O}}$ is reference evapotranspiration.

Climatic classification based on moisture index

\begin{tabular}{ll}
\hline Moisture index value & Climatic group \\
\hline-80 & Extremely dry \\
-80 to -60 & Semi- dry \\
-60 to -40 & Dry \\
-40 to -20 & Slightly dry \\
-20 to 0 & Slightly moist \\
0 to 50 & Moist \\
50 to 100 & Wet \\
100 & Extremely wet \\
\hline
\end{tabular}

Computation of aridity index (AI)

Aridity index for different locations has been computed according to the UNESCO (1979) procedure as following:

Aridity index $(\mathrm{AI})=\frac{\text { Precipitation }(\mathrm{P})}{\text { Reference Evapotranspiration }\left(\mathrm{ET}_{\mathrm{O}}\right)}$ Climate classification based on aridity index (UNEP, 1997)

\begin{tabular}{ll}
\hline Climate class & Aridity index \\
\hline Hyper-arid & $<0.03$ \\
Arid & $0.03-0.20$ \\
Semi-arid & $0.20-0.50$ \\
Dry sub humid & $0.50-0.65$ \\
Humid & $>0.65$ \\
\hline
\end{tabular}

\section{Trend analysis}

Trends in ETo, aridity index and moisture index was studied by conducting regression analysis.

\section{RESULTS AND DISCUSSION}

\section{Variations in reference evapotranspiration}

Among the three regions, annual $\mathrm{ET}_{\mathrm{O}}$ was observed to be in the order: south-western zone (1722.9 \pm 36.9 $\mathrm{mm})>$ central zone $(1628.5 \pm 44.5 \mathrm{~mm})>$ north-east zone $(1564.9 \pm 73.7 \mathrm{~mm})$. In north-east zone, it was higher in Gurdaspur (1567.3 $\pm 61.7 \mathrm{~mm}$ ), in the central zone in Amritsar $(1681.5 \pm 47.8 \mathrm{~mm})$ and in south-western zone in Bathinda
$(1732.9 \pm 53.3 \mathrm{~mm})$, whereas the variability in annual $\mathrm{ET}_{\mathrm{O}}$ was found highest in SBS Nagar (5.8\%), Patiala (3.7 \%) and Bathinda $(3.1 \%)$ in the corresponding regions. Temporal variability conducted by regression analysis indicated a significant increase in annual $\mathrm{ET}_{\mathrm{O}}$ in the north-east region (@ 3.41 mm/year), Patiala (@ 0.10 mm/year),Firozpur (0.09 mm/year), significant decrease at Ludhiana (@-2.55 mm/ year) in central region and Bathinda (@-1.37 mm/year) in south-west region (Table 1). $\mathrm{ET}_{\mathrm{O}}$ in Gurdaspur and SBS Nagar was statistically similar to Ludhiana, but different from all other locations in central and south-west zone. Annual ET at Bathinda and Firozpur was statistically similar to Amritsar. Similarly at Ludhiana annual $\mathrm{ET}_{\mathrm{O}}$ was statistically similar to that at Gurdaspur, SBS Nagar and Patiala (Table 4).

During kharif season, $\mathrm{ET}_{\mathrm{O}}$ was observed in the order: south-west zone $(1074.9 \pm 29.9 \mathrm{~mm})>$ central $(1007.3 \pm 35.0)$ $>$ north-east zone $(988.2 \pm 44.3 \mathrm{~mm})$. In the north-east region, it was higher in Gurdaspur $(997.4 \pm 39.5 \mathrm{~mm})$, in central zone in Amritar (1056.6 $\pm 37.5 \mathrm{~mm})$ and in south-west zone in Bathinda $(1078.9 \pm 41.1 \mathrm{~mm})$, whereas the variability in kharif season $\mathrm{ET}_{\mathrm{O}}$ was observed to be highest in SBS Nagar (5.6\%), Ludhiana (4.6\%) and Bathinda (3.8\%) in the corresponding zones. Temporal variability analysis indicated a significant increase in $\mathrm{ET}_{\mathrm{O}}$ in north-east region (@ $1.81 \mathrm{~mm} /$ year), at Ludhiana (@2.32 mm/year) in the central plain zone and at Bathinda $(0.08 \mathrm{~mm} /$ year) in south-west zone (Table 1). Gurdaspur and SBS Nagar district had $\mathrm{ET}_{\mathrm{O}}$ statistically similar to Ludhiana and Patiala. Bathinda and Firozpur in the south-west zone had $\mathrm{ET}_{\mathrm{O}}$ statistically similar to Amritsar (Table 4).

Similar to annual and kharif season, $\mathrm{ET}_{\mathrm{O}}$ during rabi season was also observed in the order south-west zone (647.5 $\pm 21.5 \mathrm{~mm})>$ central $(620.6 \pm 24.5 \mathrm{~mm})>$ north-east zone $(576.3 \pm 32.0 \mathrm{~mm})$. In the north-east zone, higher $\mathrm{ET}_{\text {o }}$ was observed higher in SBS Nagar $(583.3 \pm 39.9 \mathrm{~mm})$; in central at Patiala $(628.3 \pm 29.3 \mathrm{~mm})$ and in south-west at Bathinda $(653.5 \pm 27.9 \mathrm{~mm})$, whereas variability in rabi season $\mathrm{ET}_{\mathrm{O}}$ was observed to be highest in SBS Nagar (6.8\%), Patiala (4.7\%), Bathinda $(4.3 \%)$ in the corresponding regions. Temporal variability analysis indicated a significant increase in rabi season $\mathrm{ET}_{\mathrm{O}}$ in the north-east region (@1.68 mm/year) and at Patiala (@0.74 mm/year) in the central region (Table 1). Gurdaspur and SBS Nagar in the north-east region has rabi season $\mathrm{ET}_{\mathrm{O}}$ statistically different from all other locations in central and south-west zones. In Amritsar, rabi season ET was statistically similar to Ludhiana, Patiala and Firozpur 
Table 3: Average AI in different agroclimatic zones of Punjab (1971 to 2018)

\begin{tabular}{|c|c|c|c|c|c|}
\hline $\begin{array}{l}\text { Agroclimatic } \\
\text { zones / stations }\end{array}$ & Mean \pm SD & $\mathrm{CV}$ & $\begin{array}{l}\text { Rate of Change } \\
\text { (unit year }{ }^{-1} \text { ) }\end{array}$ & $\mathrm{R}^{2}$ & Trend \\
\hline \multicolumn{6}{|c|}{ Annual AI } \\
\hline \multicolumn{6}{|l|}{ North-east zone } \\
\hline Gurdaspur & $0.7 \pm 0.20$ & 25.41 & -0.002 & 0.039 & NS \\
\hline SBS Nagar & $0.7 \pm 0.20$ & 26.36 & -0.001 & 0.01 & NS \\
\hline Average & $0.66 \pm 0.16$ & 24.24 & 0 & 0.03 & NS \\
\hline \multicolumn{6}{|c|}{ Central plain zone } \\
\hline Amritsar & $0.4 \pm 0.10$ & 31.33 & 0 & 0 & NS \\
\hline Ludhiana & $0.5 \pm 0.10$ & 31.06 & 0.001 & 0.01 & NS \\
\hline Patiala & $0.5 \pm 0.20$ & 34.02 & -0.001 & 0.00 & NS \\
\hline Average & $0.47 \pm 0.13$ & 27.66 & 0 & 0.00 & NS \\
\hline \multicolumn{6}{|c|}{ South-western zone } \\
\hline Bathinda & $0.3 \pm 0.01$ & 33.77 & 0.092 & 0.023 & NS \\
\hline Firozpur & $0.3 \pm 0.01$ & 33.49 & 0.027 & 0.002 & NS \\
\hline Average & $0.3 \pm 0.01$ & 30.77 & 0.060 & 0.01 & NS \\
\hline
\end{tabular}

\section{Kharif AI}

\section{North-east zone}

Gurdaspur

SBS Nagar

Average

\section{Central plain zone}

Amritsar

Ludhiana

Patiala

Average

South-western zone

Bathinda

Firozpur

Average

North-east zone

Gurdaspur

SBS Nagar

Average

\section{Central plain zone}

Amritsar

Ludhiana

Patiala

Average

South-western zone

Bathinda

Firozpur

Average

\section{$0.8 \pm 0.20$ \\ $0.9 \pm 0.30$}

$0.85 \pm 0.24$

$0.6 \pm 0.22$

$0.6 \pm 0.30$

$0.7 \pm 0.30$

$0.62 \pm 0.19$

$0.3 \pm 0.10$

$0.4 \pm 0.10$

$0.35 \pm 0.12$

$0.4 \pm 0.20$

$0.3 \pm 0.20$

$0.34 \pm 0.17$

$0.2 \pm 0.10$

$0.2 \pm 0.10$

$0.2 \pm 0.10$

$0.2 \pm 0.11$

$0.1 \pm 0.10$

$0.11 \pm 0.06$

$0.11 \pm 0.05$

$\begin{array}{llll}30.09 & -0.002 & 0.01 & \text { NS } \\ 29.44 & 0 & 0 & \text { NS } \\ 28.24 & 0 & 0.01 & \text { NS }\end{array}$

38.96

0.230

$0.02 \quad \mathrm{NS}$

39.34

0.001

$0.01 \quad \mathrm{NS}$

39.00

0

$0.00 \quad \mathrm{NS}$

30.65

0

$0.01 \quad \mathrm{NS}$

36.57

0.002

$0.03 \quad$ NS

36.06

0.001

$0.01 \quad \mathrm{NS}$

34.29

0

0.02

NS

\section{Rabi AI}

52.18

54.07

50.00

59.24

56.69

69.70

55.00

51.79

50.19

45.45
$-0.003$

$-0.003$

0

$-0.063$

$-0.001$

$-0.001$

0

0.00

$-0.001$

0
$0.051 \quad \mathrm{NS}$

$0.054 \quad \mathrm{NS}$

$0.06 \quad \mathrm{NS}$

$0.004 \quad \mathrm{NS}$

$0.01 \quad \mathrm{NS}$

0.02 NS

$0.01 \quad \mathrm{NS}$

$0.011 \quad$ NS

$0.05 \quad \mathrm{NS}$

$0.03 \quad \mathrm{NS}$ 
Table 4: Mean difference and analysis of significance (P values) of variability in $\mathrm{ET}_{\mathrm{O}}$

\begin{tabular}{|c|c|c|c|c|c|c|c|}
\hline & \multicolumn{2}{|c|}{ North-east region } & \multicolumn{3}{|c|}{ Central region } & \multicolumn{2}{|c|}{ South-west region } \\
\hline & Gurdaspur & SBS nagar & Amritsar & Ludhiana & Patiala & Bathinda & Firozpur \\
\hline \multicolumn{8}{|c|}{ Annual ET } \\
\hline Gurdaspur & - & $4.71(1.00)$ & $-114.29(0.00)$ & $-25.08(0.347)$ & $-44.23 *(0.004)$ & $-129.97(0.000)$ & $-144.68 *(0.000)$ \\
\hline SBS nagar & $-4.72(1.000)$ & - & $-119.0(0.000)$ & $-29.81(0.159)$ & $-48.95 *(0.001)$ & $-134.69 *(0.000)$ & $-149.4 *(0.000)$ \\
\hline \multicolumn{8}{|c|}{ Central region } \\
\hline Ludhiana & $25.09(0.347)$ & $29.81(0.159)$ & $-89.2 *(0.000)$ & - & $19.14(0.674)$ & $-104.88 *(0.000)$ & $-119.59 *(0.000)$ \\
\hline Patiala & $44.23 *(0.004)$ & $48.95 *(0.001)$ & $-70.05 *(0.000)$ & $19.14(0.674)$ & & $-85.73 *(0.000)$ & $-100.4 *(0.000)$ \\
\hline \multicolumn{8}{|c|}{ South-western region } \\
\hline Bathinda & $129.97 *(0.000)$ & $134.69 *(0.000)$ & $15.68(0.842)$ & $104.88 *(0.000)$ & $85.74 *(0.000)$ & - & $-14.71(0.878)$ \\
\hline
\end{tabular}

\section{Kharif ET}

North-east region

\begin{tabular}{|c|c|c|c|c|c|c|c|}
\hline Gurdaspur & - & $18.47(0.29)$ & $-59.12 *(0.00)$ & $15.07(0.543)$ & $14.55 *(0.585)$ & $-53.17 *(0.000)$ & $-73.16 *(0.000)$ \\
\hline SBS nagar & $-18.47(0.29)$ & - & $-77.58 *(0.00)$ & $-3.39(1.00)$ & $-3.91(0.999)$ & $-71.63 *(0.00)$ & $-91.62 *(0.000)$ \\
\hline \multicolumn{8}{|c|}{ Central region } \\
\hline Amritsar & $59.11 *(0.00)$ & $77.58 *(0.00)$ & - & $74.19 *(0.00)$ & $73.67 *(0.00)$ & $5.94(0.992)$ & $-14.04(0.627)$ \\
\hline Ludhiana & $-15.07(0.543)$ & $3.39(1.00)$ & $-74.19 *(0.00)$ & - & $0.51(1.00)$ & $-68.24 *(0.00)$ & $-88.23 *(0.00)$ \\
\hline Patiala & $14.56 *(0.585)$ & $3.92(0.999)$ & $-73.67 *(0.00)$ & $0.51(1.00)$ & - & $-67.72 *(0.00)$ & $-87.72 *(0.00)$ \\
\hline \multicolumn{8}{|c|}{ South-western region } \\
\hline Bathinda & $53.17 *(0.000)$ & $71.63 *(0.000)$ & $-5.94(0.992)$ & $68.24 *(0.00)$ & $67.72 *(0.00)$ & - & $-19.99(0.202)$ \\
\hline Firozpur & $73.16 *(0.000)$ & $91.62 *(0.000)$ & $14.04(0.627)$ & $88.23 *(0.000)$ & $87.71 *(0.000)$ & $19.99(0.202)$ & - \\
\hline
\end{tabular}

Rabi ET

\begin{tabular}{llllllll} 
North-east region & & & & & & \\
Gurdaspur & - & $-14.65(0.127)$ & $-54.3 *(0.00)$ & $-39.48(0.000)$ & $-59.04 *(0.000)$ & $-76.6 *(0.000)$ & $-70.6 *(0.000)$ \\
SBS nagar & $-14.65(0.127)$ & - & $-39.59 *(0.00)$ & $-24.82 *(0.00)$ & $-44.38(0.000)$ & $-61.91 *(0.000)$ & $-55.93 *(0.000)$ \\
Central region & & & & & & & \\
Amritsar & $54.25 *(0.00)$ & $39.59 *(0.000)$ & - & $14.76(0.121)$ & $-4.78(0.979)$ & $-22.31(0.002)$ & $-16.34(0.059)$ \\
Ludhiana & $39.48 *(0.000)$ & $24.82(0.000)$ & $-14.77(0.121)$ & - & $-19.55(0.010)$ & $-37.08 *(0.000)$ & $-31.11 *(0.000)$ \\
Patiala & $59.04 *(0.000)$ & $44.38(0.000)$ & $4.78(0.979)$ & $19.55 *(0.010)$ & - & $-17.52(0.032)$ & $-11.55(0.382)$ \\
South-western region & & & & & & & \\
Bathinda & $76.56 *(0.000)$ & $61.91 *(0.000)$ & $-22.31 *(0.002)$ & $37.08 *(0.000)$ & $17.52 *(0.032)$ & - & - \\
Firozpur & $70.59 *(0.000)$ & $55.93 *(0.000)$ & $16.34(0.059)$ & $31.11 *(0.000)$ & $11.55(0.382)$ & $-5.97(0.938)$ & - \\
\hline
\end{tabular}

and at Patiala also it was statistically similar to Amritsar and Firozpur (Table 4). Maximum ET $_{\mathrm{O}}$ in South-western zone of Punjab might be due to its prevailing 'arid' climate in this zone (Kingra et al. 2017).

\section{Variations in moisture index (MI)}

Among the three regions, annual MI was observed to be in the order north-east zone $(-33.82 \pm 15.68)>$ central zone $(-53.49 \pm 13.15)>$ south-western zone $(-74.23 \pm 7.97)$. In north-east zone, it was higher in SBS Nagar (-32.5 \pm 17.8$)$, in central zone in Ludhiana (-52.1 \pm 14.9$)$ and in south-western zone in Firozpur $(-73.6 \pm 8.8)$, whereas the variability in annual MI was found to be highest in Gurdaspur (47.1\%), Amritsar (25.4\%) and Bathinda (11.3\%) in the corresponding zones. Analysis of temporal variability didn't indicate any significant trend (Table 2). Annual MI within districts of a 
Table 5: Mean difference and analysis of significance ( $\mathrm{P}$ values) of variability in $\mathrm{MI}$

\begin{tabular}{|c|c|c|c|c|c|c|c|}
\hline & \multicolumn{2}{|c|}{ North-east region } & \multicolumn{3}{|c|}{ Central region } & \multicolumn{2}{|c|}{ South-western region } \\
\hline & Gurdaspur & SBS nagar & Amritsar & Ludhiana & Patiala & Bathinda & Firozpur \\
\hline \multicolumn{8}{|c|}{ Annual MI } \\
\hline \multicolumn{8}{|c|}{ North-east region } \\
\hline Gurdaspur & - & $-2.55(0.975)$ & $20.02 *(0.000)$ & $-17.02 *(0.000)$ & $-18.12 *(0.000)$ & $39.7 *(0.000)$ & $38.54 *(0.000)$ \\
\hline SBS nagar & $-2.55(0.975)$ & - & $-22.58 *(0.000)$ & $-19.57 *(0.000)$ & $-20.68 *(0.00)$ & $-42.26 *(0.000)$ & $-41.10 *(0.000)$ \\
\hline \multicolumn{8}{|c|}{ Central region } \\
\hline Amritsar & $-20.02 *(0.000)$ & $-22.58 *(0.000)$ & - & $-3.00(0.946)$ & $1.900(0.995)$ & $19.68 *(0.000)$ & $-18.51 *(0.000)$ \\
\hline Ludhiana & $-17.02 *(0.000)$ & $19.57(0.000)$ & $-3.00(0.946)$ & - & $-1.10(1.000)$ & $-22.68 *(0.000)$ & $-21.51 *(0.000)$ \\
\hline Patiala & $-18.12 *(0.000)$ & $-20.68 *(0.000)$ & $1.9(0.995)$ & $-1.10(1.000)$ & - & $21.58 *(0.000)$ & $-20.4 *(0.000)$ \\
\hline \multicolumn{8}{|c|}{ South-western region } \\
\hline Bathinda & $-39.71 *(0.000)$ & $-42.26 *(0.000)$ & $-19.68 *(0.000)$ & $-22.68 *(0.000)$ & $-21.58 *(0.000)$ & - & $-1.16(1.000)$ \\
\hline Firozpur & $-38.54 *(0.000)$ & $-41.10 *(0.000)$ & $-18.51 *(0.000)$ & $-21.51 *(0.000)$ & $-20.41 *(0.00)$ & $-1.16(1.000)$ & 0 \\
\hline
\end{tabular}

\section{Kharif MI}

North-east region

Gurdaspur -

SBS nagar $\quad 7.37(0.656)$

\begin{tabular}{|c|c|c|c|c|c|}
\hline$-7.37(0.656)$ & $25.3 *(0.00)$ & $17.51 *(0.002)$ & $15.03 *(0.016)$ & $47.61 *(0.000)$ & $-45.92 *(0.000)$ \\
\hline & $-32.71 *(0.000)$ & $24.89 *(0.000)$ & $22.41 *(0.000)$ & $54.9 *(0.000)$ & $53.30 *(0.000)$ \\
\hline
\end{tabular}

Central region

Amritsar $\quad-25.3 *(0.000) \quad-32.7 *(0.000) \quad-$

Ludhiana $\quad-17.51(0.002) \quad-24.89(0.000) \quad 7.81(0.591)$

Patiala $\quad-15.03 *(0.016) \quad-22.41 *(0.000)$

$10.29(0.252)$

$24.89 *(0.000)$

$22.41 *(0.000)$

$54.9 *(0.000)$

$53.30 *(0.000)$

South-western region

\begin{tabular}{llllllll} 
Bathinda & $-47.61 *(0.000)$ & $-54.99 *(0.000)$ & $-22.27 *(0.000)$ & $-30.09 *(0.00)$ & $-32.57 *(0.00)$ & - & $-1.69(1.000)$ \\
Firozpur & $45.92 *(0.000)$ & $53.30 *(0.000)$ & $-20.5 *^{*}(0.000)$ & $-28.40 *(0.000)$ & $30.88 *(0.000)$ & $1.69(1.000)$ & - \\
\hline
\end{tabular}

\section{Rabi MI}

\begin{tabular}{|c|c|c|c|c|c|c|c|}
\hline \multicolumn{8}{|c|}{ North-east region } \\
\hline Gurdaspur & - & $4.76(0.535)$ & $12.35 *(0.000)$ & $14.91 *(0.000)$ & $20.50 *(0.000)$ & $24.94 *(0.000)$ & $-24.77 *(0.000)$ \\
\hline SBS nagar & $-4.76(0.535)$ & - & $-7.59 *(0.060)$ & $-10.15 *(0.002)$ & $-15.74 *(0.000)$ & $-20.18 *(0.00)$ & $-20.01 *(0.000)$ \\
\hline \multicolumn{8}{|c|}{ Central region } \\
\hline Amritsar & $-12.35 *(0.000)$ & $-7.59(0.060)$ & - & $2.56(0.958)$ & $8.14 *(0.032)$ & $-12.59 *(0.000)$ & $12.42 *(0.000)$ \\
\hline Ludhiana & $-14.91 *(0.000)$ & $-10.15^{*}(0.002)$ & $-2.56(0.958)$ & - & $-5.58(0.334)$ & $10.02 *(0.003)$ & $-9.86 *(0.004)$ \\
\hline Patiala & $-20.50 *(0.000)$ & $-15.74 *(0.000)$ & $-8.14 *(0.032)$ & $-5.58(0.334)$ & - & $4.44(0.617)$ & $4.27(0.659)$ \\
\hline \multicolumn{8}{|c|}{ South-western region } \\
\hline Bathinda & $-24.94 *(0.000)$ & $-20.18 *(0.000)$ & $-12.59 *(0.000)$ & $-10.02 *(0.003)$ & $-4.44(0.617)$ & - & $-0.165(1.000)$ \\
\hline Firozpur & $-24.7 *(0.000)$ & $-20.01 *(0.000)$ & $-12.42 *(0.000)$ & $-9.86 *(0.004)$ & $-4.27(0.659)$ & $0.16(1.000)$ & - \\
\hline
\end{tabular}

zone was statistically similar but different from districts in other zones (Table 5).

During kharif season, MI was observed to be in the order: North-east zone $(-14.7 \pm 23.67)>$ central zone $(-37.7 \pm 18.82)$ $>$ south-west zone $(-65.15 \pm 11.7)$. In north-east zone, it was higher in SBS Nagar $(-11 \pm 26.2)$, in central zone in Patiala $(-33.4 \pm 26)$ and in south-west zone in Bathinda (-66.0 \pm 12.5$)$, whereas the variability in kharif MI was found to be highest in Gurdaspur (133.5\%), Amritsar (50.12\%), Bathinda (18.95\%) in the corresponding regions. Temporal variability analysis didn't indicate significant trend (Table 2). kharif MI within districts of a zone was statistically similar but different from districts in other zones (Table 5).

During rabi season, MI was observed to be in the order: 
Table 6: Mean difference and analysis of significance (P values) of variability in AI

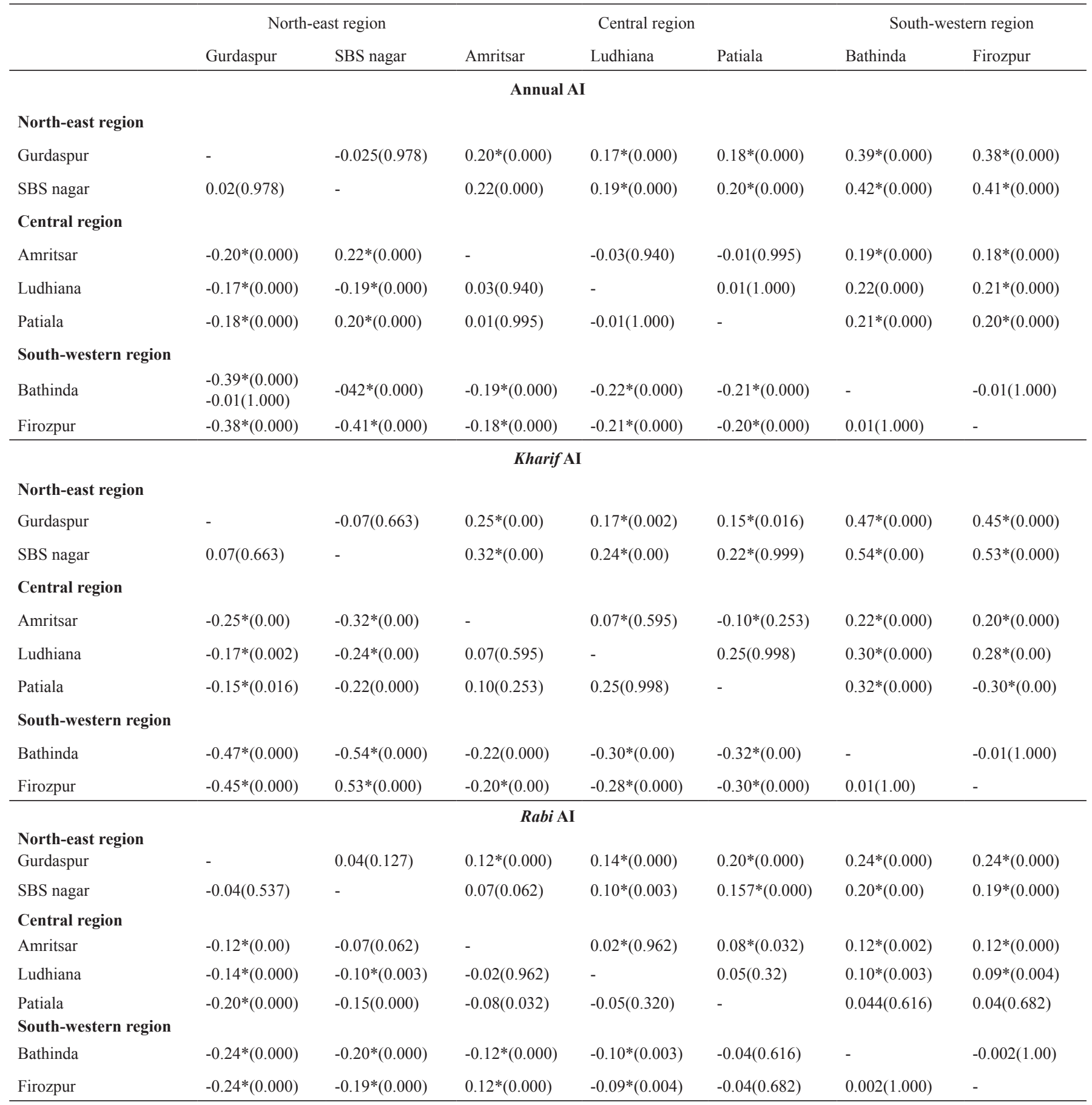

North-east zone $(-66.2 \pm 16.51)>$ central zone $(-79.96 \pm 11.01)$ $>$ south-west zone $(-89.0 \pm 5.25)$. In north-east zone, it was higher in Gurdaspur (-63.9 \pm 18.8$)$, in central zone in Amritsar $(-76.3 \pm 14.1)$ and in south-west zone there was no difference

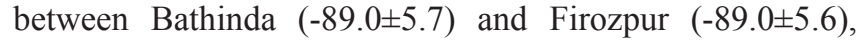
whereas the variability in rabi MI was found to be highest in SBS Nagar (-24.75\%), Patiala (-12.63\%), Firozpur (-6.24\%) in the corresponding regions. Analysis of temporal variability didn’t indicate any significant trend (Table 2). Rabi MI in
Amritsar was statistically similar to SBS Nagar and Ludhiana. Similarly, rabi MI in Patiala was statistically similar to Ludhiana in central zone and Bathinda and Ferozpur in south-west zone. Likewise, rabi MI in Bathinda and Firozpur was statistically similar to Patiala in central zone (Table 5).

\section{Variations in aridity index (AI)}

Among the three regions, annual AI was observed in the order: North-east zone $(0.66 \pm 0.16)>$ central zone 
$(0.47 \pm 0.13)>$ south-west zone $(0.3 \pm 0.01)$ (Table 3$)$. In northeast zone it didn't vary significantly between Gurdaspur and SBS Nagar, in Central it was higher in Ludhiana and Patiala and in south-west zone also there was no significant difference between Bathinda and Ferozpur, whereas variability in annual AI was observed to be highest in SBS Nagar (26.3\%), Patiala (34.02\%) and Bathinda (33.7\%) in the corresponding zones. Analysis of temporal variability didn't indicate any significant trend Annual AI within districts of a zone was statistically similar but different from districts in other zones (Table 6).

During kharif season, AI was observed to be in the order: North-east zone $(0.85 \pm 0.24)>$ central zone $(0.62 \pm 0.19)$ $>$ south-west zone $(0.35 \pm 0.12)$. In north-east zone, it was higher in SBS Nagar $(0.9 \pm 0.3)$, in central zone in Patiala $(0.7 \pm 0.3)$ and in south-west zone in Firozpur $(0.4 \pm 0.1)$, whereas the variability in kharif AI was found to be highest in Gurdaspur (30.09\%), Ludhiana (39.34\%), Bathinda $(36.57 \%)$ in the corresponding regions. Analysis of temporal variability didn't indicate any significant trend (Table 3 ). Mean difference of AI among different districts and analysis of their significance indicated that kharif AI within districts of a zone was statistically similar but different from districts in other zones (Table 6).

During rabi season, AI was observed to be in the order: North-east zone $(0.340 .17 \pm)>$ central zone $(0.20 .11 \pm)$ $>$ south-west zone $(0.110 .05 \pm)$. In north-east zone, it was higher in Gurdaspur (0.40.2 \pm ), in central zone it was similar in all the three locations viz. Amritsar, Patiala and Ludhiana $(0.20 .1 \pm)$ and in south-west zone in Firozpur (0.110.06 \pm ), whereas the variability in rabi AI was found to be highest in SBS Nagar (54.07\%), Patiala (69.7\%), Bathinda (51.79\%) in the corresponding regions. Analysis of temporal variability didn't indicate any significant trend (Table 5). Rabi AI in SBS nagar was statistically similar to Amritsar. Similarly, rabi AI in Patiala was statistically similar to Ludhiana in central zone and Bathinda and Ferozpur in south-west zone. Likewise, rabi AI in Bathinda and Firozpur was statistically similar to Patiala in central zone (Table 6).

\section{CONCLUSION}

The study concluded that large spatio-temporal variability in $\mathrm{ET}_{\mathrm{O}}$, $\mathrm{MI}$ and $\mathrm{AI}$ warns about the critical situation of moisture availability in the region as the water resources are already over-exploited thus warning about dire need of water management technologies to sustain water resources and crop productivity in the region.

\section{REFERENCES}

Hargreaves, G. H. and Samani, Z. A. (1985). Reference crop evapotranspiration from ambient air temperature. American Society of Agricultural Engineers (Microfiche collection) (USA). fiche no 85-2517. United Nations Educational, Scientific and Cultural Organization (UNESCO) (1979) Map of the world distribution of arid region: Map at scale 1:25,000,000 with explanatory note. MAB Technical Notes 7, UNESCO, Paris.

Halikatti, S.I., Potdar, M.P., Hiremath, S.M. and Dineshkumar, S.P. (2010). Annual and seasonal rainfall variability at Dharwad, Karnataka. J. Agrometeorol. 12 (1): 136-37.

Holzmann, M.E., Rivas, R. and Piccolo, M. C. (2013). Estimating soil moisture and the relationship with crop yield using surface temperature and vegetation index. Int. J. App. Earth Obs. Geo. 12:181-192.

Kaur, J. and Gill, K. K. (2017). Seasonal variation in evapotranspiration for rice and wheat crop seasons during different time slices under climate change scenarios in central Punjab. J. Agrometeorol. 19: $150-155$.

Kingra, P. K., Setia, R., Singh, S., Kaur, J., Kaur, S., Singh, S. P., Kukal, S. S. and Pateriya, B. (2017). Climatic variability and its characterisation over Punjab, India. J. Agrometeorol. 19 (3): 246-250.

Krishnan, A. and Singh, M. (1972). Soil climate zones in relation to cropping patterns. Proceedings of the Symposium on Cropping Patterns in India, ICAR, New Delhi. 172 - 85 .

Mao, J., Fu, W., Shi, X., Ricciuto, D. M., Fisher, J. B., Dickinson, R. E., Wei, Y., Shem, W., Piao, S. and Wang, K. (2015). Disentangling climatic and anthropogenic controls on global terrestrial evapotranspiration trends. Environ. Res. Lett. 10(9): 094008.

Singh, S. and Park, J. (2018). Drivers of change in groundwater resources: a case study of the Indian Punjab. Food Sec., 10:965-979.

United Nations Educational, Scientific and Cultural Organization (UNESCO), (1979). Map of the world distribution of arid region: Map at scale 1:25,000,000 with explanatory note. MAB Technical Notes 7 , UNESCO, Paris.

UNEP (United Nations Environment Programme). (1997). World atlas of desertification 2ED. UNEP, London. 